\title{
Editorial: International ESA Conference on Guidance, Navigation and Control Systems 2017
}

\author{
S. Bennani ${ }^{1} \cdot$ A. Benoit ${ }^{1} \cdot$ G. Ortega ${ }^{2} \cdot$ S. Theil $^{3}$
}

Published online: 28 November 2018

(c) CEAS 2018

This special issue of the CEAS Space Journal has been prepared from a selection of the most interesting presentations delivered throughout different sessions of the 10th International ESA Conference on Guidance, Navigation and Control Systems.

Every 3 years, this conference offers a unique window into the most recent experiences and innovations of the international GNC community, as well as a detailed insight into the demanding requirements of future missions. The latest ESA GNC conference, held from 29 May to 2 June 2017 in Salzburg, was attended by 275 engineers, managers and decision makers from around the globe. A broad range of GNC-related topics was covered by presentations of the highest caliber.

Thanks to the authors who accepted to submit their papers to the CEAS Space Journal and thanks to the reviewers, we are happy to provide a representative and up-to-date survey covering challenging missions and associated GNC developments, advanced techniques for hybrid optical navigation, adaptive augmented control or failure management and the most recent development in the area of CMOS Active Pixel Sensors for Star Trackers.

A. Benoit

alain.benoit@esa.int

S. Bennani

samir.bennani@esa.int

G. Ortega

guillermo.ortega@esa.int

S. Theil

stephan.theil@dlr.de

1 GNC, AOCS and Pointing Division, ESA/ESTEC, Keplerlaan 1, 2201 AZ Noordwijk, ZH, The Netherlands

2 Propulsion and Aerothermodynamics Division, ESA/ESTEC, Keplerlaan 1, 2201 AZ Noordwijk, ZH, The Netherlands

3 Institute of Space Systems, DLR, Robert Hooke Str. 7, 28359 Bremen, Germany
Two cutting-edge drag-free missions, namely LISA Pathfinder and Microscope, are presented in the first two papers. Having been launched respectively in December 2015 and April 2016 for a 2 year mission, they have provided unique in-flight results and lessons learnt. The first one is a technology demonstrator mission paving the way for the gravitational wave observatory LISA. The second one is a scientific mission dedicated to the test of the Equivalence Principle with unprecedented accuracy.

The next two papers present two very different missions coping with high pointing accuracy and stability requirements. GOES-16, the first spacecraft of the next-generation geostationary weather satellites, implements an active vibration damping (AVD) system which has been successfully tuned in-orbit through model identification. ATHENA, a High Energy Astrophysics mission currently in early feasibility assessment phase, is triggering a number of techniques including pointing with a hexapod as line-of-sight actuator in the control loop.

Planetary exploration missions are also highly inspirational and demanding in terms of GNC-enabling techniques, some of them being shared with in-orbit servicing missions. This is the case of rendezvous and capture, with a paper presenting the combined use of chaser GNC and robotic arm controller for proximity operations. Autonomy is then addressed by the next paper in the specific case of a deep space mission towards asteroids.

The next three papers cover advanced GNC and failure management techniques for specific applications: lunar robotic missions with pin-point landing requirements, launch vehicles with adaptive augmenting control and conventional satellites with autonomous management of reaction wheels failures. 
This special issue finally presents FaintStar, the new generation of Active Pixel Sensors developed by ESA for Star Trackers, which integrates significant logic functionality with the pixel array, aiming at an imaging system-on-chip.

Last but not least, special acknowledgements are due to the CEAS Space Journal reviewers and to the GNC, AOCS and Pointing Division in charge of the International ESA GNC Conference in the Directorate of Technology, Engineering and Quality of the European Space Agency. 